\title{
Heterogeneous expression of zinc-finger E-box-binding homeobox 1 plays a pivotal role in metastasis via regulation of miR-200c in epithelial-mesenchymal transition
}

\author{
YUTA MUTO $^{1}$, KOICHI SUZUKI ${ }^{1}$, TAKAHARU KATO ${ }^{1}$, SHINGO TSUJINAKA ${ }^{1}$, KOSUKE ICHIDA ${ }^{1}$, \\ YUJI TAKAYAMA $^{1}$, TARO FUKUI ${ }^{1}$, NAO KAKIZAWA ${ }^{1}$, FUMIAKI WATANABE $^{1}$, MASAAKI SAITO ${ }^{1}$, \\ KAZUSHIGE FUTSUHARA ${ }^{1}$, HIROSHI NODA ${ }^{1}$, YASUYUKI MIYAKURA ${ }^{1}$, \\ FUMIO KONISHI $^{2}$ and TOSHIKI RIKIYAMA ${ }^{1}$
}

${ }^{1}$ Department of Surgery, Saitama Medical Center, Jichi Medical University, Omiya-ku, Saitama 330-8503;

${ }^{2}$ Nerima-Hikarigaoka Hospital, Nerima-ku, Tokyo 179-0072, Japan

Received March 25, 2016; Accepted May 18, 2016

DOI: 10.3892/ijo.2016.3583

\begin{abstract}
Although epithelial-mesenchymal transition (EMT) has been implicated as the pivotal event in metastasis, there is insufficient evidence related to EMT in clinical settings. Intratumor heterogeneity may lead to underestimation of gene expression representing EMT. In the present study, we investigated the expression of EMT-associated genes and microRNAs in primary colorectal cancer while considering intratumor heterogeneity. One-hundred and thirty-three multiple spatially separated samples were obtained from 8 patients with metastatic colorectal cancers and 8 with non-metastatic colorectal cancers, from the tumor center (TC), invasive front (IF) and metastasis. Differences in gene and microRNA expression were investigated by microarray and quantitative reversetranscription PCR. Gene expression microarray analysis detected 7920 sites showing differing levels of gene expression among the TC, IF and metastasis. Expression of the EMT-associated gene zinc-finger E-box-binding homeobox 1 (ZEB1) significantly increased in the IF $(\mathrm{P}<0.01)$. To exclude individual differences, the expression ratio between TC and IF in each tumor was applied to analysis. This approach enabled recognition of the activation of the VEGF and Wnt signaling pathways, which were involved in metastasis via promotion of EMT. While no activation of these pathways was seen at the $\mathrm{TC}$, regardless of whether tumors were metastatic or non-metastatic, they were preferentially activated at the IF in metastatic tumors, where high ZEB1 expression was seen in connection with decreased miR-200c expression. Multiple sampling in a tumor revealed that heterogeneous ZEB1 expression induced
\end{abstract}

Correspondence to: Dr Koichi Suzuki, Department of Surgery, Saitama Medical Center, Jichi Medical University, 1-847 Amanuma-cho, Omiya-ku, Saitama 330-8503, Japan

E-mail: ksuzbnhm@yahoo.co.jp

Key words: colorectal cancer, epithelial-mesenchymal transition, intratumor heterogeneity, miR-200, ZEB1, metastasis by EMT-associated signaling pathways played a pivotal role in metastasis via regulation of miR-200c.

\section{Introduction}

Colorectal cancer is one of the most common cancers worldwide and has good prognosis if detected early. However, metastasis of colorectal cancer causes cancer-related deaths. Increasing evidence suggests that epithelial-mesenchymal transition (EMT) plays an important role in tumor progression and metastasis formation in several types of cancers, including colorectal cancer (1-4). EMT is a biologic process that enables a polarized epithelial cell to undergo several biochemical changes. This biological process enables epithelial cells to acquire the mesenchymal cell phenotype, including enhanced migratory ability and invasiveness (5). During this process, epithelial cells lose E-cadherin expression and exhibit loss of cell-cell adhesion, reorganization of the cytoskeleton by switching from keratin to vimentin intermediate filaments, loss of apical-basal polarity, acquisition of a fibroblast-like cell shape and increase in motility $(6,7)$.

Many molecular targets are involved in EMT. Some key transcription factors, including SNAIL, zinc-finger E-box-binding homeobox (ZEB), and basic helix-loop-helix transcription factors, are considered to play central roles in EMT $(2,4)$. These molecules can directly bind the promoter region of the E-cadherin gene and inhibit E-cadherin transcription (8). Consequently, tumor cells exhibit mesenchymal features and obtain abilities for migration and invasiveness. Activation of these molecules is triggered by many signaling pathways such as the TGF $\beta$ superfamily, Wnt, Notch, VEGF, epidermal growth factor (EGF) and hypoxia-inducible factor pathways $(9,10)$. These signaling pathways are involved in carcinogenesis via the mutual influence of the tumor and components of its microenvironment on the dynamic control of EMT (2). microRNAs also participate in the regulation of EMT by binding to mRNAs, and then microRNAs control the translation or degradation of mRNAs $(11,12)$. EMT allows individual cells to delaminate from primary tumors and migrate 
Table I. Patient characteristics.

\begin{tabular}{lccccccccl}
\hline Patient & Gender & Age & Location & T & N & M & UICC-stage & $\begin{array}{c}\text { Histologic } \\
\text { type }\end{array}$ & $\begin{array}{c}\text { Distant } \\
\text { metastasis }^{\mathrm{c}}\end{array}$ \\
\hline 1 & M & 65 & A & T3 & 0 & 0 & II A & Well & No \\
2 & M & 51 & R & T3 & 0 & 0 & II A & Well & No \\
3 & M & 79 & S & T4b & 0 & 0 & II C & Well & No \\
4 & M & 70 & S & T4a & 0 & 0 & II B & Well & No \\
5 & M & 57 & R & T3 & 2 & $1 \mathrm{a}$ & IV a & Well & Liver \\
6 & F & 64 & S & T4a & 2 & $1 \mathrm{a}$ & IV a & Mode & Liver \\
7 & F & 76 & T & T4a & 1 & $1 \mathrm{~b}$ & IV b & Mode & Liver, per, ovary \\
8 & F & 61 & S & T4a & 2 & $1 \mathrm{~b}$ & IV b & Mode & Per, ovary \\
9 & F & 73 & R & T2 & 0 & 0 & I & Well & No \\
10 & F & 77 & A & T3 & 0 & 0 & II A & Well & No \\
11 & F & 78 & T & T4a & 1 & 0 & III B & Well & No \\
12 & F & 46 & S & T3 & 1 & 0 & III B & Well & No \\
13 & F & 86 & A & T4a & 2 & $1 \mathrm{a}$ & IV a & Muc & Lung \\
14 & M & 78 & A & T4a & 2 & $1 \mathrm{a}$ & IV a & Well & Liver \\
15 & M & 72 & R & T3 & 1 & $1 \mathrm{a}$ & IV a & Mode & Distant lymph nodes \\
16 & F & 41 & S & T4a & 1 & $1 \mathrm{a}$ & IV a & Well & Liver
\end{tabular}

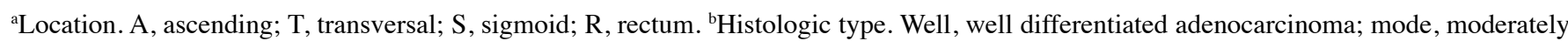
differentiated adenocarcinoma; muc, mucinous adenocarcinoma. ${ }^{~}$ Distant metastasis. Per, per itoneal dissemination.

along the extracellular matrix network $(10,13)$. These processes are observed in vitro, but not in vivo; in vitro studies are often subject to criticism because the stromal interactions and tumor environment in vivo cannot be replicated in vitro $(6,9,14,15)$. There is little evidence of EMT in human tumors due to the great diversity in cellular organization $(13,15,16)$. Tumors accumulate somatic aberrations through an evolutionary process (16), inducing heterogeneous features in a tumor; this is known as intratumor heterogeneity. Multiple genetically distinct subclones have been detected within a primary tumor (17-19). Improvements in technologies, including nextgeneration sequencing, have enabled in-depth verification of intratumor heterogeneity at the molecular level. In clear cell renal cell carcinoma, tumor subclones within the primary tumor appear geographically distinct $(18,19)$. Intratumor heterogeneity in other tumor types has also been noted and has been reported to influence targeted therapy and polygenic drug resistance (20). Therefore, intratumor heterogeneity should be considered while studying EMT-associated genes.

In the present study, we obtained multiple spatially separated samples from primary colorectal carcinomas and their metastatic sites to elucidate the heterogeneous molecular profiles of EMT. We investigated the distribution of EMT-associated gene expression and activation of EMT-associated signaling pathways and microRNAs and compared gene expression between the tumor center (TC) and invasive front (IF) of metastatic and non-metastatic tumors.

\section{Materials and methods}

Patients and tissues. The colorectal cancer tissue samples were obtained from 8 patients who had metastatic colorectal cancer and 8 patients who had non-metastatic colorectal cancer; these 16 patients had undergone surgery from December 2013 to December 2014 at the Saitama Medical Center, Jichi Medical University, Japan. The present study was approved by the Research Ethics Committee at Jichi Medical University. Written informed consent was obtained from each study participant. Table I displays the clinical and histopathological characteristics of the patients.

Multiple spatially separated samples were taken from the TC or IF in each tumor. The TC was defined as an area with a depth of 5-10 mm from the surface of the tumor. The IF was defined as an area 1-3 mm away from the boundary between the tumor and muscle, serosa, or other organs (Fig. 1). Tissue samples were obtained from metastasis areas in 6 patients and normal mucosa in 11 patients (Table II).

Both normal and tumor tissues were obtained immediately after surgery, immersed in RNAlater (Ambion, Inc., Austin, TX, USA), and stored at $-80^{\circ} \mathrm{C}$ in our laboratory until processing. RNA isolated from samples obtained from 38 sites of 8 tumors were used for microarray analysis and RNA obtained from 133 sites of 16 tumors were used for quantitative real-time reverse-transcription (RT) PCR (RT-qPCR). Eighty-eight samples obtained from the TC and IF of 8 tumors that had sufficient sample volume for obtaining microRNA were used for the microRNA analysis. Formalin-fixed paraffin embedded (FFPE) samples were also obtained from the abovementioned 16 patients.

RNA extraction. Total RNA was extracted from samples using the Illustra RNAspin Mini RNA Isolation kit (GE Healthcare UK, Buckinghamshire, UK) and the miRCURY RNA Isolation kit (Exiqon, Vedbaek, Denmark) according to 
Table II. Characteristics of samples.

\begin{tabular}{|c|c|c|c|c|c|c|}
\hline Patient & Normal colon tissue & Tumor center & Invasive front & Metastasis & Other & Subtotal \\
\hline 1 & 0 & 1 & 1 & 0 & - & 2 \\
\hline 2 & 0 & 1 & 2 & 0 & - & 3 \\
\hline 3 & 0 & 2 & 3 & 0 & - & 5 \\
\hline 4 & 0 & 5 & 3 & 0 & - & 8 \\
\hline 5 & 1 & 1 & 2 & 1 & - & 5 \\
\hline 6 & 1 & 4 & 2 & 1 & - & 8 \\
\hline 7 & 0 & 1 & 2 & 1 & - & 4 \\
\hline 8 & 1 & 4 & 3 & 3 & - & 11 \\
\hline 9 & 1 & 6 & 3 & 0 & - & 10 \\
\hline 10 & 1 & 5 & 1 & 0 & - & 7 \\
\hline 11 & 1 & 4 & 4 & 0 & - & 9 \\
\hline 12 & 1 & 3 & 3 & 0 & Lymph node & 8 \\
\hline 13 & 1 & 6 & 6 & 1 & - & 14 \\
\hline 14 & 1 & 11 & 3 & 4 & - & 19 \\
\hline 15 & 1 & 5 & 6 & 0 & - & 12 \\
\hline 16 & 1 & 5 & 2 & 0 & - & 8 \\
\hline Subtotal & 11 & 64 & 46 & 11 & 1 & 133 \\
\hline
\end{tabular}

Table III. Primers for gene expression assay and micro RNA assays used in the present study.

\begin{tabular}{lcc}
\hline Target & Product number & Manufacturer \\
\hline TaqMan ${ }^{\circledR}$ Gene Expression assays & & Applied Biosystems \\
ZEB1 & Hs00232783_m1 & Applied Biosystems \\
ZEB2 & Hs00207691_m1 & Applied Biosystems \\
SNAI1 & Hs00195591_m1 & Applied Biosystems \\
CDH1 & Hs01023894_m1 & Applied Biosystems \\
GAPDH & Hs03929097_g1 & Exiqon \\
microRNA LNA ${ }^{\text {TM }}$ PCR primers & & Exiqon \\
hsa-miR-200a-3p & 204707 & Exiqon \\
hsa-miR-200b-3p & 206071 & Exiqon \\
hsa-miR-200c-3p & 204482 & Exiqon \\
hsa-miR-141-3p & 204504 & 204488 \\
hsa-miR-423-3p & & \\
\hline
\end{tabular}

the manufacturer's instructions. For assessing RNA quality and yield, $\mathrm{A}_{260} / \mathrm{A}_{280}$ and $\mathrm{A}_{260} / \mathrm{A}_{230}$ ratios for RNA preparation samples were analyzed with a Nano-Drop ${ }^{\circledR}$ ND-1000 spectrophotometer (NanoDrop Technologies, Inc., Wilmington, DE, USA) and RNA integrity number (RIN) was calculated by an Agilent 2100 Bioanalyzer (Agilent Technologies, Santa Clara, CA, USA) (21). RNAs with RINs of 7-10 were used for microarray and RT-qPCR.

Microarray expression profiling. The gene expression microarray experiments were performed using Agilent SurePrint G3 Human Gene Expression 8x60K ver2.0 (Agilent Technologies) according to the manufacturer's instructions (One-Color Microarray-Based Gene Expression Analysis Protocol Version Jan. 2012; Agilent Technologies). In brief, cyanine-3 (Cy3)-labeled cRNA was prepared from 75 ng RNA using the One-Color Low Input Quick Amp labeling kit (Agilent Technologies), followed by purification with the RNeasy Mini kit (Qiagen, Hilden, Germany). Dye incorporation and cRNA yield were checked with the NanoDrop spectrophotometer. For hybridization, $600 \mathrm{ng}$ of Cy3-labeled cRNA was fragmented at $60^{\circ} \mathrm{C}$ for $30 \mathrm{~min}$. On completion of the fragmentation reaction, $25 \mu \mathrm{l}$ of $2 \mathrm{X}$ Agilent hybridization buffer was added to the fragmentation mixture and hybridized for $17 \mathrm{~h}$ at $65^{\circ} \mathrm{C}$ in a rotating Agilent hybridization oven. After hybridization, microarrays were washed with GE Wash Buffer. Slides were scanned after washing on the Agilent Technologies Microarray scanner. The fluorescence intensities on scanned images were extracted and preprocessed by Agilent Feature Extraction software (v10.7.3.1). The raw signals were normalized using the percentile shift normalization method; the value was set at 75 th percentile and log transformed. Universal Human Reference 

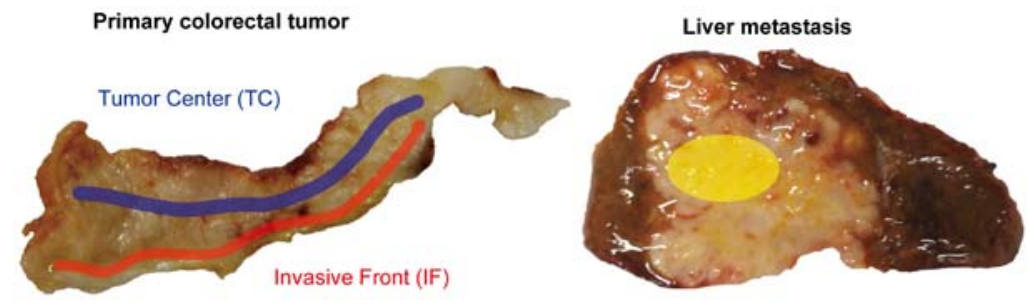

Figure 1. Sample collections. Multiple spatially separated samples were obtained from the tumor center (blue line), invasive front (red line) and metastasis (yellow circle)

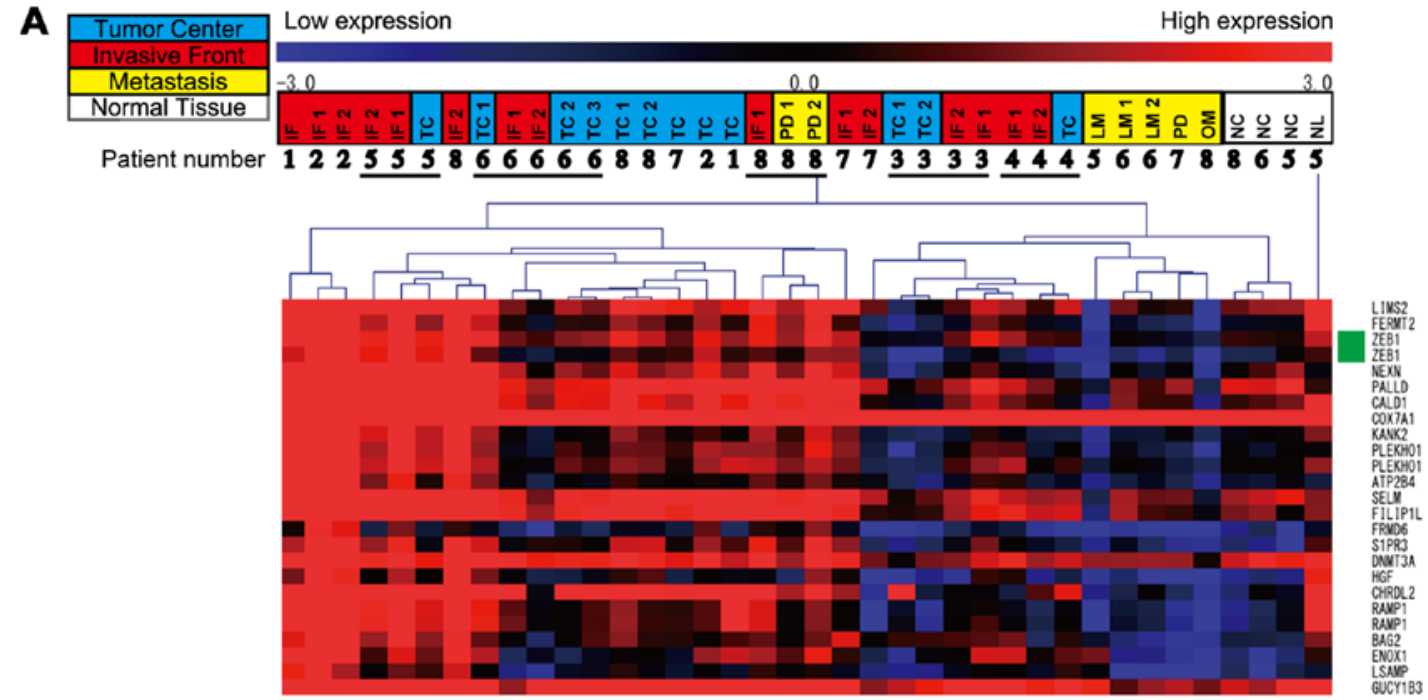

B
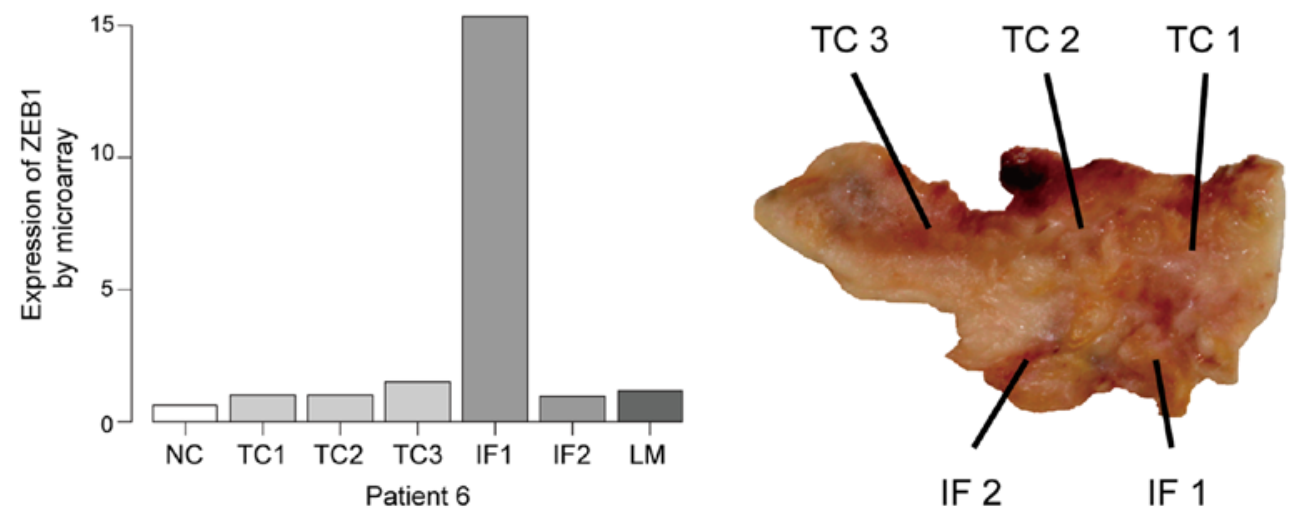

IF 2

IF 1

Figure 2. Gene expression microarray analysis. (A) Clustering of the samples according to their expression profile. High expression is indicated in red. Low expression is indicated in blue. Samples are shown at the top of the heatmap. Colors in upper row indicate the following: tumor center in light blue, invasive front in red, metastasis in yellow. Patient number is indicated in the lower row. On the right side of the heatmap, the genes associated with gene expression array probes are shown. ZEB1 probes are indicated in green. Clustering was performed by complete linkage using Pearson's correlation on a subset of array probes previously selected by ANOVA. A part of the heatmap is displayed in this figure. (B) Left panel showed the expression of ZEB1 of the tumor in patient 6 by gene expression microarray. Right panel display the sampling area of the tumor in patient 6 . TC, tumor center; IF, invasive front; $\mathrm{NC}$, normal colon mucosa; LM, liver metastasis; NL, normal liver tissue; OM, ovarian metastasis; PD, peritoneal dissemination.

RNA (Agilent Technologies) was used as the control. Data have been deposited in Gene Expression Omnibus (accession number GSE75117).

RT-qPCR assay for mRNA expression. RT was performed using the High Capacity RNA-to-cDNA kit (Applied Biosystems, Carlsbad, CA, USA). RT-qPCR assays were performed using TaqMan ${ }^{\circledR}$ Gene Expression Assays (Applied Biosystems; Table III) and TaqMan ${ }^{\circledR}$ Gene Expression Master Mix on the QuantStudio ${ }^{\text {TM }}$ 12K Flex Real-Time PCR system
(Applied Biosystems). Thermal cycling conditions were as follows: $95^{\circ} \mathrm{C}$ for $10 \mathrm{~min}$, followed by 40 cycles of $95^{\circ} \mathrm{C}$ for $15 \mathrm{sec}$ and $60^{\circ} \mathrm{C}$ for $1 \mathrm{~min}$. The expression level of each gene was determined using the fluorescence intensity measurements from the QuantStudio ${ }^{\mathrm{TM}}$ 12K Flex Data Analysis software. A GAPDH fragment was amplified as an internal control. RT-qPCR assays were repeated two times.

$R T-q P C R$ assay for miR-200 expression. Total RNA (10 ng) extracted with the use of the miRCURY RNA Isolation kit 
Table IV. Results of gene annotation and pathway ontology.

\begin{tabular}{|c|c|c|c|}
\hline Term & Count & P-value & Benjamini \\
\hline Axon guidance & 18 & 4.3E-4 & $3.4 \mathrm{E}-2$ \\
\hline Endocytosis & 16 & $6.5 \mathrm{E}-2$ & $4.3 \mathrm{E}-1$ \\
\hline VEGF signaling pathway & 14 & $1.4 \mathrm{E}-4$ & $2.1 \mathrm{E}-2$ \\
\hline $\mathrm{T}$ cell receptor signaling pathway & 14 & $4.5 \mathrm{E}-3$ & $1.1 \mathrm{E}-1$ \\
\hline Wnt signaling pathway & 14 & $5.8 \mathrm{E}-2$ & $4.1 \mathrm{E}-1$ \\
\hline ErbB signaling pathway & 13 & $2.0 \mathrm{E}-3$ & $7.7 \mathrm{E}-2$ \\
\hline Neurotrophin signaling pathway & 13 & $3.2 \mathrm{E}-2$ & $3.1 \mathrm{E}-1$ \\
\hline Tight junction & 13 & $5.2 \mathrm{E}-2$ & $4.1 \mathrm{E}-1$ \\
\hline Pancreatic cancer & 11 & $4.5 \mathrm{E}-3$ & $1.3 \mathrm{E}-1$ \\
\hline B cell receptor signaling pathway & 11 & $6.0 \mathrm{E}-3$ & $1.3 \mathrm{E}-1$ \\
\hline Fc epsilon RI signaling pathway & 11 & 7.9E-3 & $1.5 \mathrm{E}-1$ \\
\hline Fc gamma R-mediated phagocytosis & 11 & $2.9 \mathrm{E}-2$ & $3.0 \mathrm{E}-1$ \\
\hline Leukocyte transendothelial migration & 11 & $9.7 \mathrm{E}-2$ & $5.1 \mathrm{E}-1$ \\
\hline Chronic myeloid leukemia & 10 & $1.7 \mathrm{E}-2$ & $2.4 \mathrm{E}-1$ \\
\hline Prostate cancer & 10 & $4.6 \mathrm{E}-2$ & $3.9 \mathrm{E}-1$ \\
\hline Progesterone-mediated oocyte maturation & 9 & $8.6 \mathrm{E}-2$ & $4.8 \mathrm{E}-1$ \\
\hline Linoleic acid metabolism & 8 & $5.1 \mathrm{E}-4$ & $2.7 \mathrm{E}-2$ \\
\hline Non-small cell lung cancer & 8 & $2.3 \mathrm{E}-2$ & $2.7 \mathrm{E}-1$ \\
\hline Glycerophospholipid metabolism & 8 & $6.8 \mathrm{E}-2$ & $4.3 \mathrm{E}-1$ \\
\hline Renal cell carcinoma & 8 & $7.8 \mathrm{E}-2$ & $4.6 \mathrm{E}-1$ \\
\hline Bladder cancer & 7 & 2.3E-2 & $2.8 \mathrm{E}-1$ \\
\hline Endometrial cancer & 7 & $5.6 \mathrm{E}-2$ & $4.2 \mathrm{E}-1$ \\
\hline Metabolism of xenobiotics by cytochrome P450 & 7 & $9.8 \mathrm{E}-2$ & $5.0 \mathrm{E}-1$ \\
\hline Dorso-ventral axis formation & 6 & $9.1 \mathrm{E}-3$ & $1.5 \mathrm{E}-1$ \\
\hline
\end{tabular}

was reverse transcribed in $10-\mu 1$ reactions by using the miRCURY LNA ${ }^{\mathrm{TM}}$ Universal cDNA Synthesis kit II (Exiqon). RT-qPCR assays for microRNAs were performed with specific microRNA LNA PCR primers (Exiqon; Table III) and ExiLENT SYBR ${ }^{\circledR}$ Green Master Mix (Exiqon). All RT-qPCR reactions were carried out in 96-well plates with the ROX Reference Dye (Applied Biosystems) in the QuantStudio ${ }^{\mathrm{TM}}$ 12K Flex Real-Time PCR System (Applied Biosystems). Thermal cycling conditions were as follows: $95^{\circ} \mathrm{C}$ for $10 \mathrm{~min}$; 40 cycles of $95^{\circ} \mathrm{C}$ for $10 \mathrm{sec}$ and $60^{\circ} \mathrm{C}$ for $1 \mathrm{~min}$; and $95^{\circ} \mathrm{C}$ for $15 \mathrm{sec}, 60^{\circ} \mathrm{C}$ for $1 \mathrm{~min}$, and $95^{\circ} \mathrm{C}$ for $15 \mathrm{sec}$. A miR-423-3p primer was used as the reference microRNA.

Immunohistochemistry. Immunohistochemistry (IHC) staining was performed on FFPE samples according to standard procedures. In summary, FFPE samples on slides were baked for $30 \mathrm{~min}$ at $58^{\circ} \mathrm{C}$, deparaffinized in xylene, rehydrated through graded alcohol, and antigen-retrieved at $98^{\circ} \mathrm{C}$ in a water bath for $30 \mathrm{~min}$ in sodium citrate buffer $(10 \mathrm{mM}$ sodium citrate, $\mathrm{pH}$ 6.0). Then, the slides were immersed in $0.3 \%$ hydrogen peroxide diluted in methanol for $20 \mathrm{~min}$ to block endogenous peroxidase activity and preincubated with $5 \%$ bovine serum albumin (BSA) at $15-20^{\circ} \mathrm{C}$ for $15 \mathrm{~min}$ to reduce non-specific reaction. Subsequently, the slides were incubated with anti-ZEB1 (\#NBP1-05987; Novus Biologicals, Littleton, CO, USA, 1:500 dilution, $4^{\circ} \mathrm{C}$ overnight), anti-ZEB2 (\#HPA003456; Sigma, St. Louis, MO, USA, 1:400 dilution, $4^{\circ} \mathrm{C}$ overnight) or anti-VEGF (\#ab46154; Abcam, Cambridge, UK, 1:200 dilution, $15-20^{\circ} \mathrm{C}$ for $30 \mathrm{~min}$ ). The slides were sequentially incubated with a secondary antibody, Histofine ${ }^{\circledR}$ Simple Stain $^{\text {TM }}$ MAX PO MULTI (Nichirei Biosciences Inc., Tokyo, Japan), at $15-20^{\circ} \mathrm{C}$ for $30 \mathrm{~min}$, and stained with DAB (Dako, Glostrup, Denmark). Finally, the slides were counterstained with Mayer's hematoxylin, dehydrated in graded concentrations of ethanol and mounted.

The staining intensity of each slide for the IF was evaluated by scanning of the whole section at low (x40) and high magnification (x200) by 2 independent researchers in a blinded manner.

Statistical analysis. Continuous variable comparisons between 2 groups were performed with the Student's t-test for variables following a normal distribution, or with the non-parametric Mann-Whitney-Wilcoxon test for variables that did not follow a normal distribution. To determine the significant genes from multiple samples, analysis of variance (ANOVA) was carried out using $\mathrm{MeV}$, using which hierarchical clustering sample and gene trees were also drawn simultaneously (22). The level 

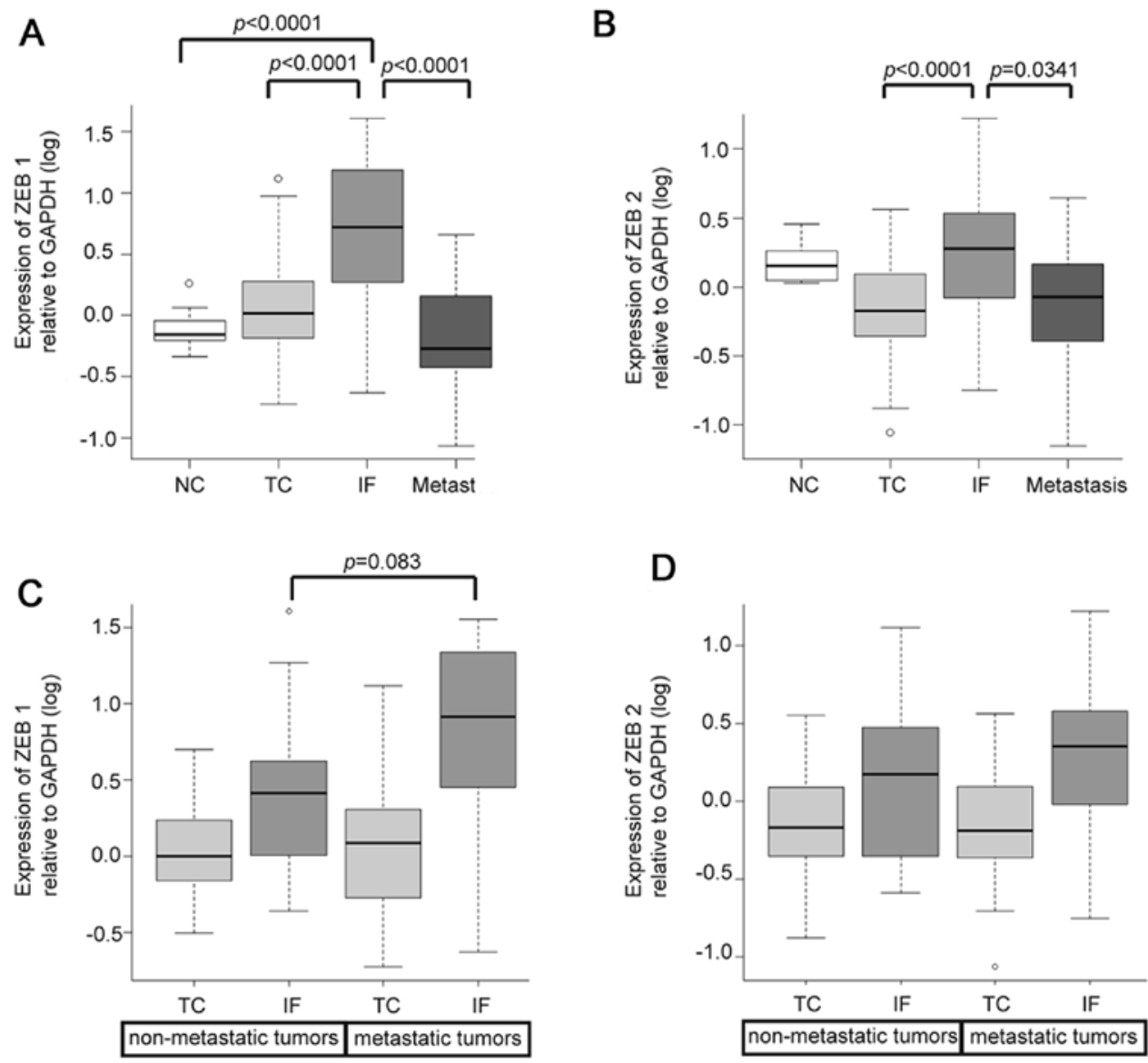

Figure 3. Expression levels of ZEB genes according to their locations and metastatic status. (A and B) Expression levels of ZEB1 (A) and ZEB2 (B) in normal colon mucosa (NC), tumor center (TC), invasive front (IF), and metastasis. (C and D) Expression levels of ZEB1 (C) and ZEB2 (D) in metastatic or nonmetastatic tumors according to their locations. Expression levels were analyzed by RT-qPCR with a $\log 2$ scale. Horizontal lines within the boxes indicate the median value. Error bars represent the standard deviation. P-values were calculated by one-way ANOVA followed by Tukey's HSD multi-hypothesis testing correction.

of statistical significance was set at $\mathrm{P}<0.05$, unless otherwise specified. Statistical analyses were performed with EZR (Saitama Medical Center, Jichi Medical University, Saitama, Japan), a graphical user interface for R 2.13.0 (R Foundation for Statistical Computing, Vienna, Austria) (23). More precisely, EZR is a modified version of $\mathrm{R}$ commander (version 1.6-3), designed to add statistical functions used frequently in biostatistics.

\section{Results}

Heterogeneous expression in EMT-associated genes and activation of the VEGF and WNT signaling pathways. Unsupervised hierarchical clustering was performed to elucidate distinct gene profiles among 3 locations, including the IF, TC and metastasis, but no clear differences were seen (data not shown). We then used ANOVA to determine whether there was location-associated gene expression; the results revealed that 7920 probes (13\% of whole probes) exhibited significant differences in levels of gene expression among 3 locations. The IF in tumors displayed higher expression of ZEB1, an EMT-associated gene, than that seen in the TC and metastasis $(\mathrm{P}<0.01)$ (Fig. 2A). There was no significant difference among the 3 locations with respect to the expression levels of other EMT-associated genes, including SNAIL, basic helix-loop- helix transcription factors and E-cadherin. Heterogeneous gene expression of ZEB1 was seen such that high levels of ZEB1 expression were found in a small part of the IF, whereas no increase in expression was seen in the TC (Fig. 2B). The ANOVA-constructed hierarchical tree revealed that expression patterns in several samples (nos. 3, 4, 5, 6 and 8) were influenced by individual differences rather than the differences between the TC and IF (Fig. 2A).

We then calculated the expression ratios between the TC and IF in each tumor to exclude these individual differences. These ratios were applied to the t-test comparing metastatic tumors and non-metastatic tumors. This analysis identified 1512 distinctive probes ( $2 \%$ of whole probes) according to the status of metastasis. Gene annotation and pathway ontology analyses were performed using the Database for Annotation, Visualization and Integrated Discovery (DAVID) and Kyoto Encyclopedia of Genes and Genomes (KEGG), which revealed that several genes were associated with the VEGF signaling pathway and Wnt signaling pathway (Table IV).

Comparison of gene expression of ZEB according to sample locations and the status of metastasis. We verified expression levels of EMT-associated genes, including ZEB, by RT-qPCR in a series of 133 clinical samples from 16 tumors. Expression levels of ZEB1 and ZEB2 in IF were significantly higher than 

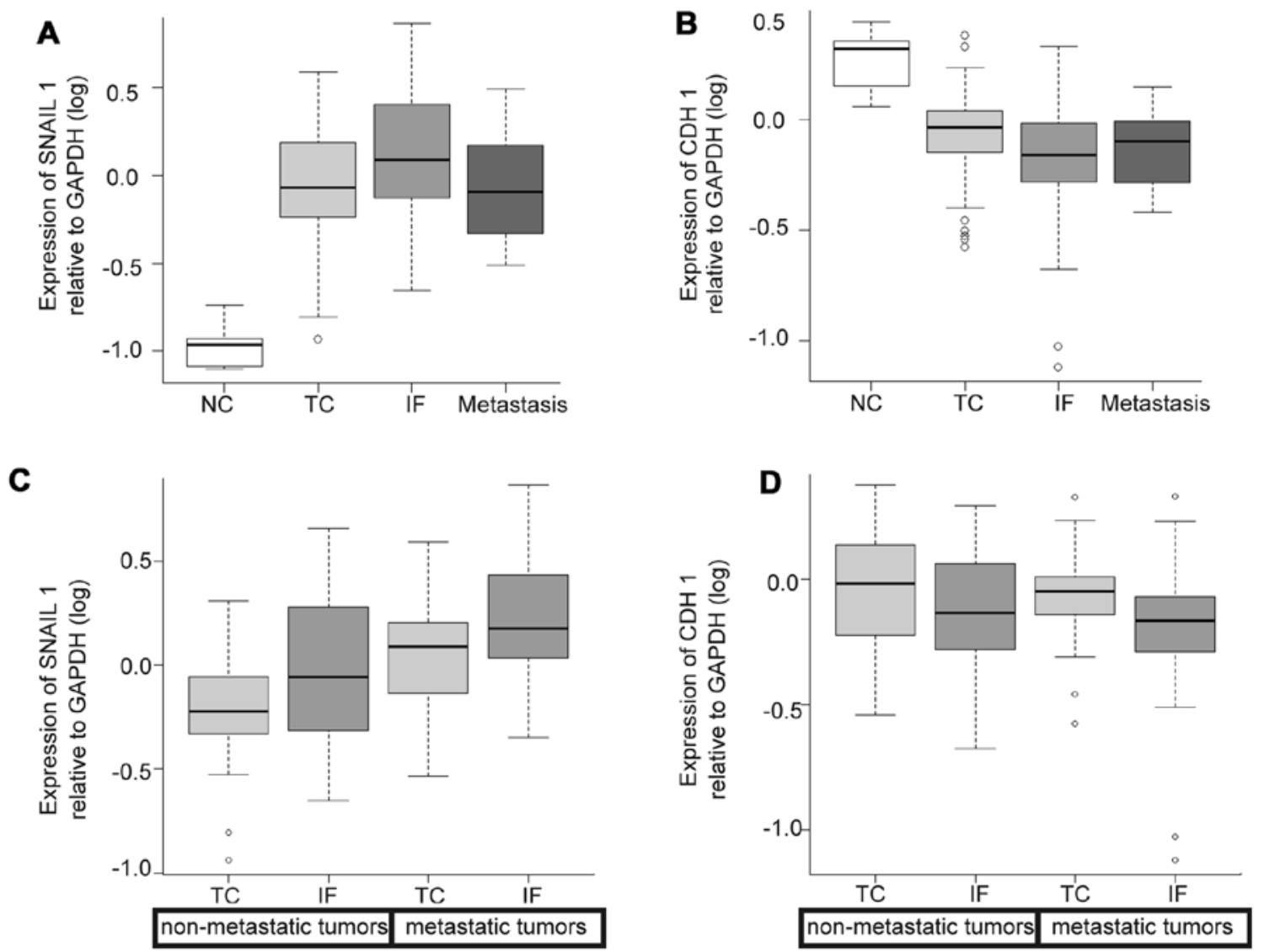

Figure 4. Expression levels of SNAIL1 and CDH1 according to their locations and metastatic status. (A and B) Expression levels of SNAIL1 (A) and E-cadherin (CDH1) (B) in normal colon mucosa (NC), tumor center (TC), invasive front (IF) and metastasis. (C and D) Expression levels of SNAIL1 (C) and E-cadherin (CDH1) (D) in metastatic or non-metastatic tumors according to their locations. Expression levels were analyzed by RT-qPCR assays with a log2 scale. Horizontal lines within the boxes indicate the median value. Error bars represent the standard deviation. P-values were calculated by one-way ANOVA followed by Tukey's HSD multi-hypothesis testing correction.

those in the TC and metastasis (Fig. 3A and B). Heterogeneous expression of ZEB proteins was ascertained by IHC. A representative sample with overexpression of ZEB1 and ZEB2 in the IF is shown in Fig. 5; ZEB1 and ZEB2 showed heterogeneous expression even in the IF. In contrast, VEGF did not display heterogeneity in the IF (Fig. 5E). Metastatic tumors preferentially expressed ZEB proteins but no statistically significant difference was found on comparing the status of metastasis.

Expression levels of SNAIL1 were higher in the IF than in the TC and metastasis, but the differences were not statistically significant. Expression of CDH1 (E-cadherin) displayed almost the same levels among the 3 locations (Fig. 4A and B).

We compared expression levels of ZEB1 and ZEB2 between metastatic tumors and non-metastatic tumors according to sample locations. Expression levels of ZEB1 in the IF tended to be higher in metastatic tumors than in non-metastatic tumors, while little variation of expression levels in TC was seen regardless of the presence or absence of metastasis (Fig. 3C). ZEB2 expression in the IF displayed little variation between metastatic tumors and non-metastatic tumors (Fig. 3D). No variation was seen in either SNAIL1 or CDH1 regardless of locations and the status of metastasis (Fig. 4C and D).

Expression levels of the miR-200 family and their correlation with ZEB. Expression levels of ZEB1 increased in metastatic tumors but the level was not significantly different from those in non-metastatic tumors. We analyzed the miR-200 family, which regulates $\mathrm{ZEB}$ genes, and measured the expression levels of this family in 88 samples. None of the variants of the miR-200 family displayed any significant difference in expression between the IF and TC regardless of the status of metastasis. When we focused on the IF for expression levels of the miR-200 family while comparing the status of metastasis, we noted lower levels of miR-200c and higher levels of miR-200a in metastatic tumors as compared to those in non-metastatic tumors; these differences were statistically significant $(\mathrm{P}=0.015$ and $\mathrm{P}=0.0065$, respectively; Fig. $6 \mathrm{~A}$ and C). None of the variants of the miR-200 family in the TC displayed any significant difference between metastatic tumors and non-metastatic tumors. Correlation analysis revealed that miR-200b and miR-200c displayed high inverse correlation with ZEB1 (Fig. 7).

\section{Discussion}

In this study, multiple spatially separated sampling in a tumor revealed heterogeneous expression of the EMT-associated gene ZEB1 and its negative regulator miR-200c. Heterogeneous expression of these genes was seen in the IF of metastatic tumors, in which EMT-inducing signal pathways such as the VEGF and Wnt pathways were activated. 

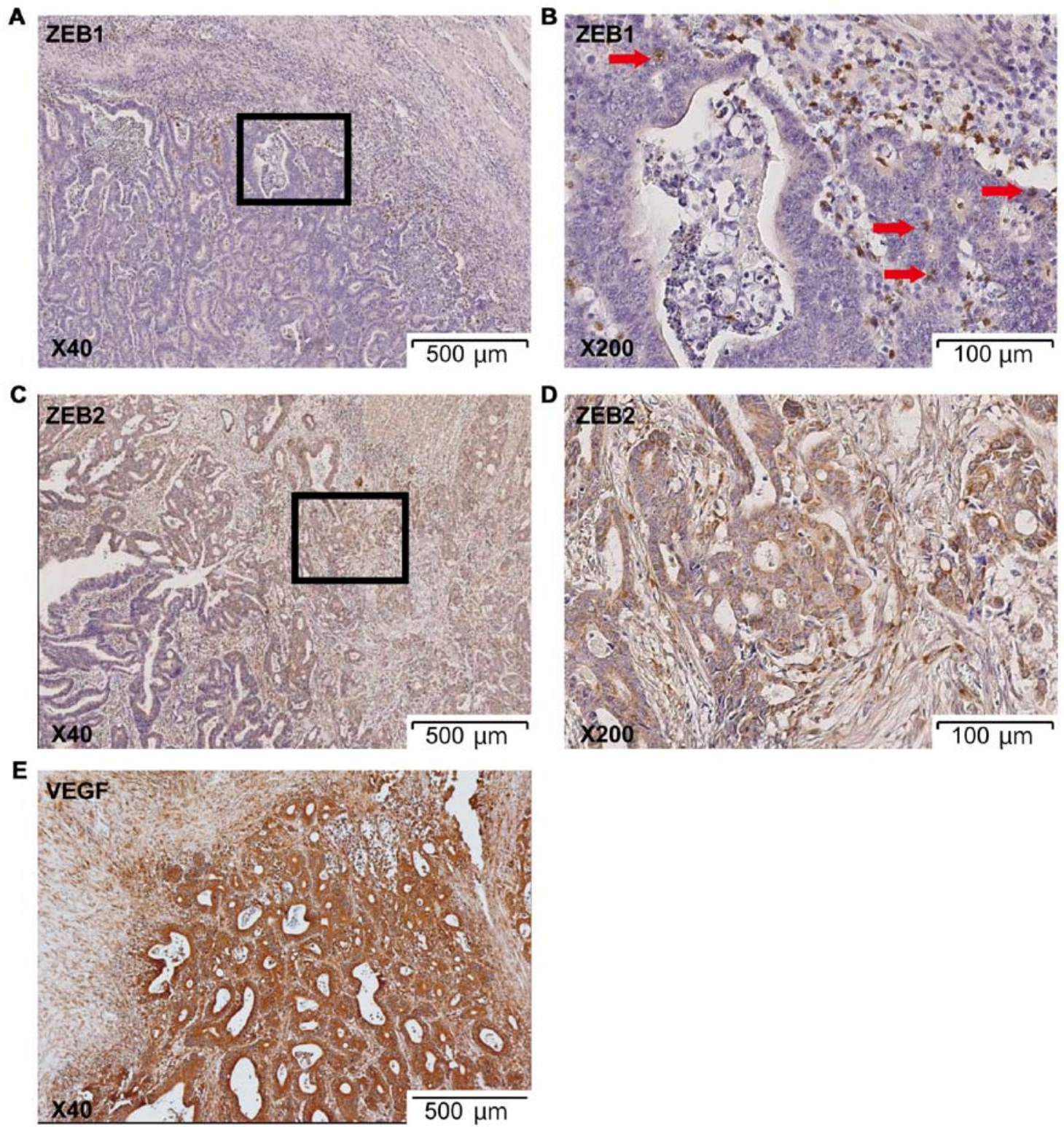

Figure 5. Immunohistochemical analysis of ZEB1, ZEB2 and VEGF. Immunohistochemical analysis of ZEB1 (A and B), ZEB2 (C and D) and VEGF (E) at the invasive front in primary colorectal cancer. A few cancer cells expressed ZEB1 (arrows). ZEB1 was predominantly expressed in the nucleus and ZEB2 was expressed in the cytoplasm. The pictures on the left were taken at x40 magnification, and boxed areas were focused and taken at x 200 magnification in the pictures on the right.

Although EMT plays a fundamental role in tumor progression and metastasis formation in vitro, evidence of EMT in human cancers has not been verified. We addressed intratumor heterogeneity of primary colorectal cancers in this study.

To investigate heterogeneous molecular profiles underlying invasion and metastasis in a tumor, we compared gene expression in the TC and IF, and the expression ratio between TC and IF was applied to the analysis. This approach enabled us to recognize the activation of some signaling pathways such as the VEGF signaling pathway and the Wnt signaling pathway (Table IV). These pathways were preferentially activated at the IF in metastatic tumors, while no activation was seen at the TC, regardless of whether the tumor was metastatic or non-metastatic. These pathways are involved in metastasis by promoting EMT. VEGFR-1 has been reported to lead to morphologic and molecular alterations in pancreatic cancer cells; these alterations are potentially similar to that of EMT to facilitate the induction of migration and invasion. In addition, VEGFR-1 activation increases the expression of EMT-associated transcription factors (24). The Wnt signaling pathway stabilizes the EMT-associated gene SNAIL by inhibition of GSK3 $\beta$ kinase (25). $\beta$-catenin is the key molecule implicated in the Wnt signaling pathway, and its activation was observed at IF in colorectal cancers through the process of EMT (26), which is consistent with our data.

Heterogeneous expression of EMT-associated genes was seen not only in an entire tumor but also in a small part of the tumor such as the IF (Fig. 2B), which indicated the importance of multiple spatially separated sampling. A single sample obtained from IF is insufficient to evaluate differences in expression levels of EMT-associated genes. Gerlinger et al $(18,19)$ stated that intratumor heterogeneity 

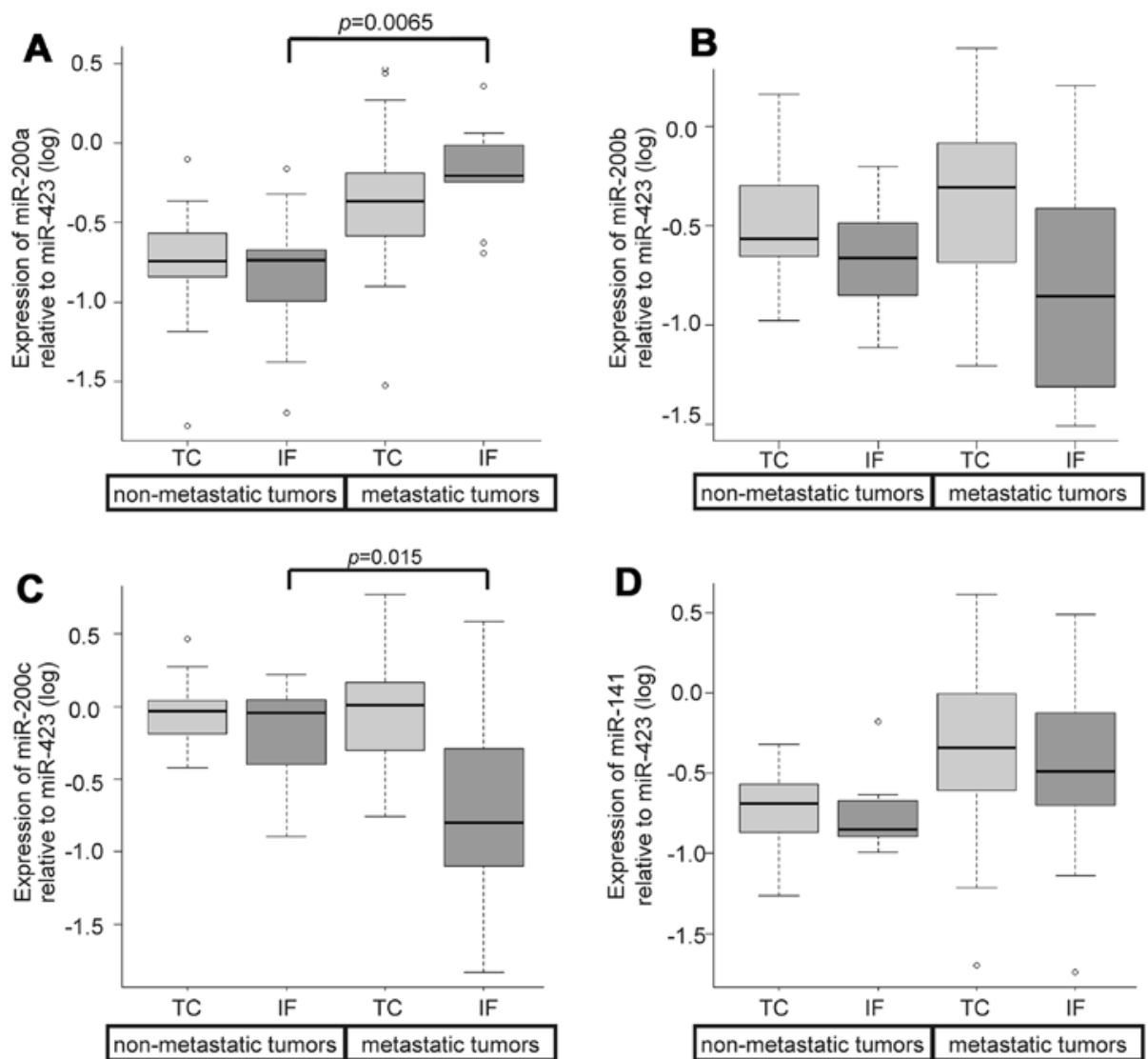

Figure 6. Expression levels of the miR-200 family according to their locations and metastatic status. Expression levels of (A) miR-200a, (B) miR-200b, (C) miR-200c and (D) miR-141 in metastatic tumors or non-metastatic tumors according to their locations. Expression levels were analyzed by RT-qPCR assays with a $\log 2$ scale. Horizontal lines within the boxes indicate the median value. Error bars represent the standard deviation. P-values were calculated by one-way ANOVA followed by Tukey's HSD multi-hypothesis testing correction.
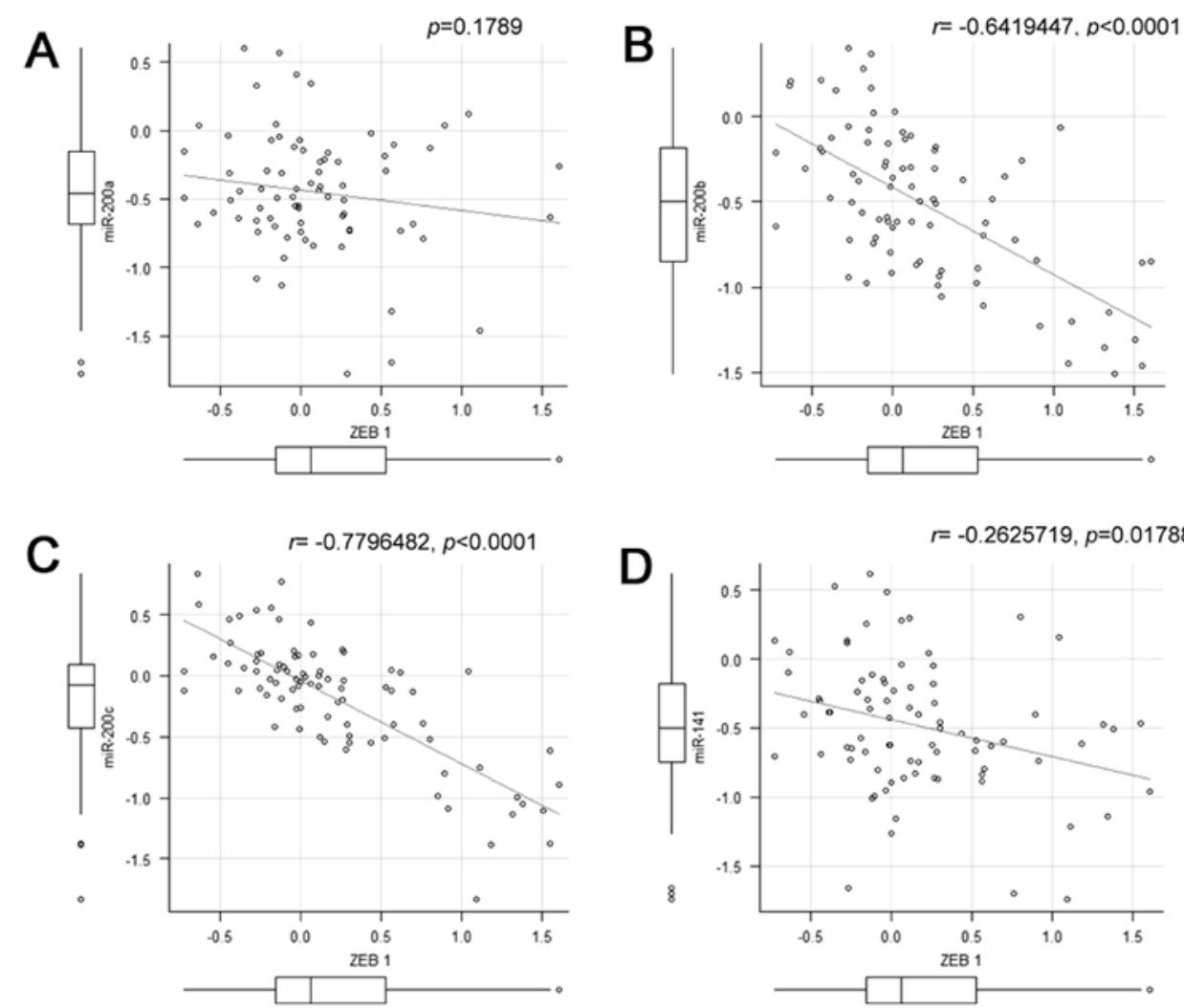

Figure 7. The correlation between ZEB1 and the miR-200 family. Dot plot of the expression levels of ZEB1 and expression levels of (A) miR-200a, (B) miR-200b, (C) miR-200c, and (D miR-141). Pearson correlation and P-value are shown in the upper right corner of each figure. 
might lead to underestimation of the genetic complexity of a tumor when single-biopsy procedures are used.

One of the EMT-associated genes, ZEB1, plays crucial roles in colorectal cancer progression (27), and high expression of ZEB1 leads to poor prognosis in primary colorectal cancer (28). However, to the best of our knowledge, no study has analyzed the relationship between ZEB-encoding genes and metastasis in primary colorectal cancers. In this study, analysis of the heterogeneous expression of ZEB1 in the IF and TC showed that the gene expression patterns for the IF differed from those for the TC. Metastatic tumors exhibited higher expression of ZEB1 at the IF than non-metastatic tumors (Fig. 3), indicating that ZEB1 was involved in metastasis. However, a statistically significant relationship between expression of ZEB1 and metastasis was not seen in the analysis.

We next focused on the microRNAs that regulate ZEB1 because microRNAs regulate multiple targets post-transcriptionally and are more stable than mRNAs in human tissue $(29,30)$. The miR-200 family is composed of five distinct microRNAs and controls EMT by downregulating the expression of the ZEB factors $(31,32)$. Among them, expression levels of miR-200c decreased at the IF in metastatic tumors. miR-200c expression showed a statistically significant difference between metastatic and non-metastatic tumors while no statistical significance was seen for ZEB1. Expression levels of miR-200c showed an inverse correlation with those of ZEB1 (Fig. 7), which is consistent with the report that miR-200 regulates the expression of ZEB1 in cultured cells (11). Considering these findings and the fact that microRNAs are stable in tissues, miR-200c would provide more useful information for predicting metastasis than ZEB1. The expression of miR-200a increased at the IF in metastatic tumors while that of miR-200c decreased (Fig. 6). This may be due to differences in structure between miR-200c and miR-200a although they have homology in their sequences (32). The expression of miR-200a did not show significant correlation with the expression of ZEB1.

In this study, heterogeneous expression of EMT-associated genes was identified by multiple spatially separated sampling. However, this procedure is too complicated and time-consuming for clinical practice. One of the ideal tools for clinical application could be 'liquid biopsy' for detecting tumor-originating genes and cells in blood; a recent study showed that miR200c could be detected in serum, which correlated with the prognosis in primary colorectal cancers (33). Although more convenient tools should be applied to clinical practice, we believe that our data obtained by multiple sampling in a tumor could provide insights into the intratumor heterogeneity of EMT-associated genes in connection with tumor metastasis in primary colorectal cancers. Tumors likely exhibit the feature of potential metastasis at the IF, in which altered expression of ZEB1 and miR-200c was seen, along with activation of EMT-inducing signaling pathways such as the VEGF and Wnt pathways.

\section{Acknowledgements}

The present study was supported in part by a grant-in-aid from The Research Award to JMU Graduate Student, JSPS
KAKENHI Grant Number 15K10147, and the JKA Foundation through its promotion funds from Keirin Racing.

\section{References}

1. Thompson EW, Newgreen DF and Tarin D: Carcinoma invasion and metastasis: A role for epithelial-mesenchymal transition? Cancer Res 65: 5991-5995, discussion 5995, 2005.

2. Peinado H, Olmeda D and Cano A: Snail, Zeb and bHLH factors in tumour progression: An alliance against the epithelial phenotype? Nat Rev Cancer 7: 415-428, 2007.

3. Eccles SA and Welch DR: Metastasis: Recent discoveries and novel treatment strategies. Lancet 369: 1742-1757, 2007.

4. Lamouille $\mathrm{S}, \mathrm{Xu} \mathrm{J}$ and Derynck R: Molecular mechanisms of epithelial-mesenchymal transition. Nat Rev Mol Cell Biol 15: 178-196, 2014.

5. Kalluri R and Weinberg RA: The basics of epithelial-mesenchymal transition. J Clin Invest 119: 1420-1428, 2009.

6. Chui MH: Insights into cancer metastasis from a clinicopathologic perspective: Epithelial-mesenchymal transition is not a necessary step. Int J Cancer 132: 1487-1495, 2013.

7. Nieto MA: Epithelial plasticity: A common theme in embryonic and cancer cells. Science 342: 1234850, 2013.

8. Nieto MA: The snail superfamily of zinc-finger transcription factors. Nat Rev Mol Cell Biol 3: 155-166, 2002.

9. Yang J and Weinberg RA: Epithelial-mesenchymal transition: At the crossroads of development and tumor metastasis. Dev Cell 14: 818-829, 2008.

10. Thiery JP, Acloque H, Huang RY and Nieto MA: Epithelialmesenchymal transitions in development and disease. Cell 139: 871-890, 2009.

11. Gregory PA, Bert AG, Paterson EL, Barry SC, Tsykin A, Farshid G, Vadas MA, Khew-Goodall Y and Goodall GJ: The miR-200 family and miR-205 regulate epithelial to mesenchymal transition by targeting ZEB1 and SIP1. Nat Cell Biol 10: 593-601, 2008.

12. Lamouille S, Subramanyam D, Blelloch R and Derynck R: Regulation of epithelial-mesenchymal and mesenchymal-epithelial transitions by microRNAs. Curr Opin Cell Biol 25: 200-207, 2013.

13. Thiery JP: Epithelial-mesenchymal transitions in tumour progression. Nat Rev Cancer 2: 442-454, 2002.

14. Busch EL, McGraw KA and Sandler RS: The potential for markers of epithelial-mesenchymal transition to improve colorectal cancer outcomes: A systematic review. Cancer Epidemiol Biomarkers Prev 23: 1164-1175, 2014.

15. Bates RC and Mercurio AM: The epithelial-mesenchymal transition (EMT) and colorectal cancer progression. Cancer Biol Ther 4: 365-370, 2005.

16. Nowell PC: The clonal evolution of tumor cell populations. Science 194: 23-28, 1976.

17. Yachida S, Jones S, Bozic I, Antal T, Leary R, Fu B, Kamiyama M, Hruban RH, Eshleman JR, Nowak MA, et al: Distant metastasis occurs late during the genetic evolution of pancreatic cancer. Nature 467: 1114-1117, 2010.

18. Gerlinger M, Rowan AJ, Horswell S, Larkin J, Endesfelder D Gronroos E, Martinez P, Matthews N, Stewart A, Tarpey P, et al: Intratumor heterogeneity and branched evolution revealed by multiregion sequencing. N Engl J Med 366: 883-892, 2012.

19. Gerlinger M, Horswell S, Larkin J, Rowan AJ, Salm MP, Varela I, Fisher R, McGranahan N, Matthews N, Santos CR, et al: Genomic architecture and evolution of clear cell renal cell carcinomas defined by multiregion sequencing. Nat Genet 46: 225-233, 2014

20. McGranahan $\mathrm{N}$ and Swanton C: Biological and therapeutic impact of intratumor heterogeneity in cancer evolution. Cancer Cell 27: 15-26, 2015.

21. Schroeder A, Mueller O, Stocker S, Salowsky R, Leiber M, Gassmann M, Lightfoot S, Menzel W, Granzow M and Ragg T: The RIN: An RNA integrity number for assigning integrity values to RNA measurements. BMC Mol Biol 7: 3, 2006.

22. Saeed AI, Sharov V, White J, Li J, Liang W, Bhagabati N, Braisted J, Klapa M, Currier T, Thiagarajan M, et al: TM4: A free, open-source system for microarray data management and analysis. Biotechniques 34: 374-378, 2003.

23. Kanda Y: Investigation of the freely available easy-to-use software 'EZR' for medical statistics. Bone Marrow Transplant 48: 452-458, 2013. 
24. Yang AD, Camp ER, Fan F, Shen L, Gray MJ, Liu W, Somcio R, Bauer TW, Wu Y, Hicklin DJ, et al: Vascular endothelial growth factor receptor-1 activation mediates epithelial to mesenchymal transition in human pancreatic carcinoma cells. Cancer Res 66: 46-51, 2006.

25. Zhou BP, Deng J, Xia W, Xu J, Li YM, Gunduz M and Hung MC: Dual regulation of Snail by GSK-3beta-mediated phosphorylation in control of epithelial-mesenchymal transition. Nat Cell Biol 6: 931-940, 2004.

26. Brabletz T, Jung A, Reu S, Porzner M, Hlubek F, KunzSchughart LA, Knuechel R and Kirchner T: Variable beta-catenin expression in colorectal cancers indicates tumor progression driven by the tumor environment. Proc Natl Acad Sci USA 98: 10356-10361, 2001.

27. Spaderna S, Schmalhofer O, Hlubek F, Berx G, Eger A, Merkel S, Jung A, Kirchner T and Brabletz T: A transient, EMT-linked loss of basement membranes indicates metastasis and poor survival in colorectal cancer. Gastroenterology 131: 830-840, 2006.

28. Peña C, García JM, Silva J, García V, Rodríguez R, Alonso I, Millán I, Salas C, de Herreros AG, Muñoz A, et al: E-cadherin and vitamin D receptor regulation by SNAIL and ZEB1 in colon cancer: Clinicopathological correlations. Hum Mol Genet 14: $3361-3370,2005$
29. Pasquinelli AE: MicroRNAs and their targets: Recognition, regulation and an emerging reciprocal relationship. Nat Rev Genet 13: 271-282, 2012.

30. Peiró-Chova L, Peña-Chilet M, López-Guerrero JA, García-Giménez JL, Alonso-Yuste E, Burgues O, Lluch A, Ferrer-Lozano J and Ribas G: High stability of microRNAs in tissue samples of compromised quality. Virchows Arch 463: 765-774, 2013.

31. Wellner U, Schubert J, Burk UC, Schmalhofer O, Zhu F, Sonntag A, Waldvogel B, Vannier C, Darling D, zur Hausen A, et al: The EMT-activator ZEB1 promotes tumorigenicity by repressing stemness-inhibiting microRNAs. Nat Cell Biol 11: 1487-1495, 2009

32. Uhlmann S, Zhang JD, Schwäger A, Mannsperger $H$, Riazalhosseini Y, Burmester S, Ward A, Korf U, Wiemann S and Sahin O: miR-200bc/429 cluster targets PLCgammal and differentially regulates proliferation and EGF-driven invasion than miR-200a/141 in breast cancer. Oncogene 29: 4297-4306, 2010.

33. Toiyama Y, Hur K, Tanaka K, Inoue Y, Kusunoki M, Boland CR and Goel A: Serum miR-200c is a novel prognostic and metastasis-predictive biomarker in patients with colorectal cancer. Ann Surg 259: 735-743, 2014 